\title{
Bases Conceptuales de la Mediación y su Importancia Actual en la Práctica Pedagógica ${ }^{1 *}$
}

\section{Conceptual Bases of the Mediation and Their Current Importance for Pedagogical Practice}

\begin{abstract}
Alberto F. Labarrere Sarduy ${ }^{2}$
Universidad Santo Tomás, Chile

(Recepción: Junio 2008 - Aceptación: Noviembre 2008)

\section{Resumen}

El presente artículo somete a análisis la mediación pedagógica, con intencionalidad de aprendizaje, en calidad de proceso central del desarrollo del sujeto. Se discute el papel de la conciencia y la intencionalidad y se reflexiona en torno a los significados que se construyen en los contextos de mediación. Se asume que la mediación, en sí misma, generalmente se hace invisible para los sujetos mediados, con lo cual se limita grandemente el propio desarrollo y su potencialidad para actuar y dominar los hechos de desarrollo. Un lugar importante se ofrece a lo que se denomina aprendizaje de la mediación. Se postula el carácter difuso y abierto de la mediación, señalándose como factor que genera su potencialidad. Se avanzan brevemente las nociones de intermediación y transmediación en calidad de aspectos susceptibles de precisión y abordajes más profundos a partir de la investigación. Por último se aborda la noción de tránsito de la ayuda a la colaboración como eje de la acción en ZDP.

Palabras clave: Mediación pedagógica, intermediación, desarrollo, enseñanza, aprendizaje.

Abstract

The present article puts under analysis the pedagogical mediation, with intentionality of learning, as central process in the development of the subject. The roll of consciousness and intentionality is discussed and some reflections are made about the meanings that are constructed in the mediation contexts. It is assumed that mediation itself becomes invisible for students which impairs development and their potentiality to acts and master the facts of development. An important place is given to what author name mediation learning. The open and fuzzy character of mediation is assumed as a factor that generates its potentiality, as well as the notions of intermediation and transmediation as aspects that must be investigated. Finally is pointed out the notion of a transit from help to collaboration as central from the action in ZDP.
\end{abstract}

Key words: Pedagogical mediation, intermediation, development, teaching, learning .

1 * Este artículo es parte de una conferencia homónima dictada en el Centro de Desarrollo Cognitivo de la Universidad Diego Portales.

2 Correspondencia a: Alberto Labarrere. Escuela de Psicología, Universidad Santo Tomás. Avenida Ejército 146, Santiago, Chile. E-mail: alabarrere@santotomas.cl 
Vamos a la mar a contemplar las olas y la puesta del sol. Acaso, también me enseñes a nadar y a pescar... Pero, mientras me enseñas a nadar y a pescar, ¿qué quieres que aprenda?: a nadar y a pescar simplemente, o cómo, por qué y para qué me enseñas a nadar y a pescar. E incluso pudieras querer que aprenda a enseñar a nadar y a pescar.

\section{(A. Labarrere)}

Autores relevantes para la psicología han aludido a la mediación con la finalidad de ofrecer ideas de sus características y resaltar su importancia para la actividad humana. Al abordar la actividad del hombre, Carl Ratner se refiere a la mediación (aunque ella no es objeto principal de su comentario) y afirma:

The difference between human and animal activity is that the animal behavior is primarily (not entirely) a biologically determined, immediate response to stimuli whereas human behave is a constructed response. ... Human biology does not establish any natural sensitivity, responsiveness, or necessary connection between the two. Instead, an inventive, constructed act mediates between stimulus and response because not biological mechanism establishes a direct, necessary stimulus-response connection. (1991, p. 14). ${ }^{3}$

Por su parte Michael Cole, hace el comentario de que la mediación:

...no es más que la interacción adecuada a la significación que ha hecho el sujeto del artefacto, en virtud de una significación compartida localmente [...]. Esto permite argumentar en primer lugar, que los “objetos” no están aislados, ellos se constituyen como tales en los contextos; y en segundo lugar, la aproximación a los artefactos, en este caso tecnológicos, está mediada por significaciones compartidas y locales construidas históricamente. El papel de la educación consiste en propiciar significaciones que permitan una relación intercultural positiva, o sea una apropiación dotada de sentido y significado, en la cual el sujeto se reconoce en la interacción como sujeto cultural activo que se recrea con aquello que la cultura le ofrece (1989, p. 128).

En la misma dirección, Feuerstein dice que:

En el corazón de la Modificabilidad Cognitiva Estructural (MCE) está la teoría de la Experiencia de Aprendizaje Mediada (EAM), a la cual atribuimos la modificabilidad humana. La EAM es una característica típica de interacción humana (énfasis A.L.), responsable de ese rasgo exclusivo de las personas que es la "modificabilidad estructural” (Noguez, 2006, en Internet).

Y, desde luego, un grande de la psicología y acaso quien mejor y más profundamente abordara la mediación en nuestro tiempo, me refiero obviamente a Vigotsky, escribió:

Sobre la conducta del hombre, en su forma general, se puede decir que su particularidad, en primer lugar está condicionada por el hecho de que el hombre interviene, activamente, en sus relaciones y, a través del medio, él mismo modifica su conducta, sometiéndola a su poder. (1987, p. 98)

Con las citas y referencias anteriores, queda bastante bien precisada la idea general de lo que

3 "La diferencia entre la actividad humana y la animal radica en que la conducta del animal es una respuesta primariamente (aunque no únicamente) determinada desde lo biológico, mientras que el comportamiento humano es una respuesta construida a los estímulos. ... La biología humana no establece sensibilidad natural alguna, tendencia a ofrecer respuestas o conexión necesaria entre ambos. Más bien ocurre que entre los estímulos y las respuestas media un acto construido, puesto que ningún mecanismo biológico establece una conexión necesaria entre las conexiones estímulo-respuesta”. (Trad. libre A.L.) 
se entiende por mediación y su importancia, e igualmente, la existencia de consenso en que es una actividad propia de los humanos por su carácter esencialmente no biológico y que se realiza mediante instrumentos, artificialmente.

Quisiera aclarar que, de acuerdo con Werstch, adopto la idea de que los procesos mediacionales pueden aparecer en al menos dos modalidades: la mediación propiamente o más propiamente semiótica y la mediación por modelación o actividad modelada; mientras que en la primera toman predominancia los símbolos, en la segunda lo hace la persona; es una mediación donde existe una interacción más o menos directa entre sujetos que interactúan mediante instrumentos, o ellos mismos se convierten en instrumentos de la interacción. Creo, además, que en la mediación pedagógica con función explícita de enseñanza y aprendizaje confluyen ambas maneras. Mi análisis no irá dirigido a la modelación semiótica, aunque esta subyace, sino a aquella situación donde el mediador es, en primera instancia, el educador o el profesor.

\section{Agenda pedagógica: de la intencionalidad y la conciencia en la mediación pedagógica.}

Obviamente, al hablar de mediación no es obligatorio hacer referencia específica a la conciencia que los sujetos tienen de los procesos mediacionales o de la propia mediación; es posible afirmar que la mayor parte de los actos y acciones mediacionales o mediadoras del hombre ocurren sin la participación de la conciencia y la intencionalidad. Pero nosotros debemos preguntarnos acerca de la importancia de la intencionalidad y la conciencia en la mediación pedagógica. Voy a partir precisamente abordando esta cuestión.

Cuando se habla de mediación pedagógica, se tiene en cuenta principalmente cierto género de acciones y actividades ejecutadas para obtener modificaciones en las personas; estas acciones que pueden ser más o menos formales, están asociadas de alguna manera y en algún momento con la intencionalidad y la conciencia de los sujetos. El objetivo de la mediación pedagógica es la transformación, desencadenar o promover procesos de reestructuración en la persona(lidad), o en los sujetos (individuales y colectivos). Por tanto, la mediación se define también por sus motivaciones, sus objetivos y consecuencias. Al estimar los efectos de los procesos mediacionales, lo mismo que al identificarlos y valorarlos hay que tener en consideración los factores antes aludidos.

El acto o la actividad mediadora, así como los procesos de mediación, tienen lugar como un entramado que se teje entre las circunstancias culturalmente definidas, la intencionalidad de los sujetos que intervienen en el contexto donde tiene lugar dicha mediación y la conciencia con que emprenden o construyen sus interacciones. De hecho, las alusiones a la mediación, realizadas por el propio Vigotsky, la definen como médula de la acción humana con su carácter instrumental. De ser así, incluso en su artificialidad, la mediación es un hecho completamente natural, que constituye el corazón de los procesos sociales y culturales. La mediación es también el eje de la culturalización de los procesos biológicos; por ejemplo, las reconocidas experiencias que realizaron Luria y Leontiev, con procesos de compensación de las funciones deterioradas haciéndolas accesibles a la conciencia. Es el caso de los enfermos que aprendieron a caminar desplazándose sobre series de pisadas o huellas estampadas en el piso. Incluso, creo ver que algo similar ocurre con la mediación instrumental propuesta por Feuerstein.

En el marco de los procesos de transformación mediada y dirigida a finalidades, la "conciencia de la mediación y de la actividad mediadora” son dos aspectos íntimamente relacionados. Ambas se expresan no sólo en el planteamiento de objetivos, sino sobre todo, en la organización y evaluación de la actividad en los espacios de interacción donde tiene lugar el desarrollo y la transformación de las personas, los instrumentos mediadores y los contextos de mediación.

Desde posiciones como las de Vigotsky y Feuerstein, la mediación tiene, explícitamente, un carácter o significado de desarrollo. Sobre todo en el caso de este último, donde la mediación instrumental se plantea como una vía para lograr el desarrollo del potencial de aprendizaje. Los sistemas de mediación propuestos por Feuerstein tienen como propósito explícito que los sujetos mejoren o desarrollen su plasticidad cognitiva y, como sabemos, esto suele conseguirse haciéndolos pasar por sistemas de "dispositivos", o instrumentos; pero sobre todo, "insertándolos” en sistemas 
de relaciones favorables a los procesos de mediación, sistemas que evocan y fomentan la plasticidad cognitiva.

Por su parte, para Vigotsky, y muchos años antes, la mediación constituye el eje del desarrollo de las funciones superiores humanas, del ser humano mismo. Puedo afirmar que el desarrollo constituye el corazón mismo de los procesos de mediación pedagógica; sin embargo, es de notar que el significado de desarrollo que implica el acto mediador en condiciones pedagógicas, por lo común queda oculto para aquellos que son mediados y cuando emerge lo hace más como una intención del mediador (en el caso de que este sea un una persona), que como un significado de lo que acontece para el sujeto mediado. Y esto es sumamente importante.

En el sentido de lo que acabo de decir, asumo que los significados que los sujetos construyen para los actos de mediación producidos en situaciones pedagógicas formalmente organizados suelen ser, fundamentalmente, significados instrumentales. Instrumentales en el sentido en que la mediación aparece como una ayuda para acometer determinada tarea, resolver cierto problema, obtener un resultado, etc., y el significado de desarrollo, a mi juicio cardinal, queda a la sombra.

La conciencia y la intencionalidad mediadora, en el sentido pedagógico están de lleno en manos de los profesores. Desde el punto de vista de los estudiantes se produce un fenómeno de invisibilidad de la mediación y mucho más de su significado de desarrollo. Este ocultamiento provoca que ellos no se impliquen o participen como actores de los procesos de mediación enfilados al desarrollo. Es algo similar a la experiencia de no mediación (Lombard y Ditton, 2008) que ha sido descrita para la realidad virtual (RV). La "ilusión de no mediación" que ha sido teorizada y comprobada prácticamente en el análisis de las situaciones de RV y que igualmente ocurre en los procesos y situaciones de mediación educacional, tiene alguna repercusión para los hechos pedagógicos en el sentido de que si para RV es importante conseguir la ilusión de no mediación, para el caso de la mediación pedagógica, enfilada hacia el aprendizaje y el desarrollo, el alto grado de invisibilidad que la acompaña, tiene efectos contraproducentes.

Durante tiempo he dicho que la enseñanza hoy escamotea muchos de los aspectos que debería mostrar a los estudiantes y trabajarlos desde la conciencia y la intencionalidad de los aprendices. Tal vez coincidamos en el hecho de que en buena medida, la enseñanza aparece ante los estudiantes como un acto de magia, donde los contornos del mundo van emergiendo poco a poco o de golpe, pero aquellos son meros espectadores de la emergencia del perfil de los objetos, de los procesos, las leyes, etc., pero no tienen acceso a los mecanismos y condiciones por los cuales y en los cuales dicho perfil emerge.

Ciertamente, la orientación constructivista, con su acento oportuno en que el mundo, el conocimiento, solo puede ser construido, por el propio sujeto, abrió una promesa que apuntaba hacia un gran paso en la consideración del sujeto del aprendizaje; mas el constructivismo se detuvo a la puerta misma de la participación activa de los estudiantes en los procesos de construcción, condenándolos a seguir viviendo en un mundo donde al construir no penetran, o lo hacen insuficiente, parcial y fragmentariamente, hacia los mecanismos que hacen posible el proceso constructivo.

Si nos situamos en un plano epistemológico, podría decirse que, como una de las consecuencias del ocultamiento de las mediaciones, se produce la separación, la escisión artificial de la enseñanza y el aprendizaje y la hieratización de los roles, responsabilidades y atribuciones de los sujetos. De hecho, enseñanza y aprendizaje son como dos mundos paralelos; desde el primero parten líneas que generan cambios en el segundo, en una relación que mucho recuerda la que establecen la causa y el efecto. Por lo común se acepta que la mediación "proviene” del profesor; incluso, en muchos países términos como "entregar" ("el profesor entrega conocimientos") y otros similares, señalan muy bien el sentido directo y apuntan a lo que realmente oculta la enseñanza.

De hecho, formalmente, los mecanismos íntimos de la actividad mediadora dada a la conciencia o en la conciencia de quienes educan en las escuelas y otras instituciones donde se forman las nuevas generaciones de ciudadanos y ciudadanas, están del lado de aquellos en quienes se ha depositado formalmente la tarea de educar. Sin embargo, podría decirse que el estudiante también genera 
mediaciones. Y estas, generalmente inadvertidas, marginadas y deslegitimadas, no reciben el debido tratamiento. No hay teoría psicológica, ni pedagógica consistente en torno a la mediación discente.

En ocasiones he afirmado que en realidad no existe la mediación. Esta, como un acto unidireccional, no tiene sentido. Toda mediación es respuesta a una mediación que la supone y provoca; se establecen cadenas o sistemas mediacionales, que constituyen la real trama del desarrollo. De hecho, el desarrollo debería ser estudiado no desde la mediación como acto unidireccional, sino como hecho de interacción. De ahí que me parezca más adecuado referirnos a la inter(mediación).

Así, la interacción educativa es más bien un hecho de intermediaciones con propósitos más o menos definidos y claros; más o menos profundos, más o menos pertinentes, consistentes y eficaces. La mediación no es acción separada de otras acciones mediadoras, sino tejidos de mediaciones, redes de acciones mediadoras que van y vienen de todas partes y hacia todas partes y que acotamos cuando especificamos actores, acciones por ellos realizadas y contextos donde se realizan.

Pero más, precisamente por lo expresado, pienso que es factible introducir el término transmediación. Igualmente, es posible afirmar que toda mediación es a la vez la historia y la promesa (futuro) de otras mediaciones. En este sentido, una acción mediadora, bajo la asunción de que sea identificable, no está potencialmente dirigida hacia un único destinatario, no es sólo sincronía; la interacción constante hace que la mediación se abra hacia el futuro y se ubique o tenga efecto más allá del "lugar imaginable", donde ella debía producirse; de hecho escapa a la intencionalidad. En cierta medida se abre y hace difusa; es anónimo eco de otras mediaciones. El carácter difuso y abierto de las acciones mediadoras y de la mediación en sí misma, genera su potencialidad. Estoy convencido de que cualquier situación o acción mediadora se extiende más allá de su contorno, se desborda a sí misma y que nunca se alcanza a percibir todas las consecuencias que de ella se derivan.

Por ejemplo, en un aula los intentos de prestar ayuda a un alumno, aunque virtualmente están orientados hacia ese alumno en particular, no tiene efectos únicamente sobre éste sino que más amplios; no sólo en la medida en que resultan procesados por otros virtuales alumnos, sino porque sus reales efectos devienen en cascada de mediaciones. Podría investigarse este fenómeno, a partir del trabajo en grupos pequeños.

De manera similar, para valorar la acción de un profesor que introduce una ayuda ante un estudiante que no comprende determinada situación, no es suficiente contar con indicadores o criterios apriorísticos de adecuación mediadora; si bien estos son necesarios, resultan insuficientes para comprender adecuadamente la acción del profesor, es también necesario remitirse a la naturaleza de la respuesta del estudiante; tras ésta es imprescindible explorar la intencionalidad de aquel. Pero más, resulta incluso una condición añadida, dirigir la atención hacia el contexto en que se genera la mediación, que a su vez constituye un elemento mediador y, entonces, juzgar acerca de la mediación o de las mediaciones en juego.

Por otro lado, dado que, como vimos, la acción mediadora está sometida a factores temporales, sus consecuencias y adecuación no pueden ser determinadas sólo en el momento en que se ejerce; para tener una idea más precisa, hay que aproximarse a la historia de la mediación, sobre todo a su función prospectiva, lo cual sólo será posible en un análisis retrospectivo.

Otro elemento que considero esencial a la hora de tratar la mediación y su importancia, se relaciona con una noción de Vigotsky, relativamente olvidada, no sé por qué. Me refiero al control de la conducta o del propio comportamiento.

Al introducir su conocida ley de la formación de las funciones superiores, Vigotsky hizo referencia al dominio de la conducta, el propio comportamiento o la actuación; vio este como un proceso mediatizado que se realiza siempre con la intervención de medios externos (mediaciones). Aunque estimo que es posible discutir los conceptos y los caminos que, según Vigotsky, sigue el logro del dominio sobre el propio comportamiento; es mi parecer que la noción es sumamente actual y presenta un considerable valor heurístico para la educación y sobre todo para el destino de los procesos de mediación pedagógica. 
Asumo que, vinculada al desarrollo, la finalidad de los procesos mediadores, sobre todo los pedagógicos, es que los sujetos alcancen el dominio de su propio comportamiento, pienso que esto puede alcanzarse de la manera más expedita y deseable, cuando se logra el dominio de la mediación, o sea cuando se producen lo que he denominado (Labarrere, 2005) “aprendizajes de la mediación”.

Cuando uno analiza lo que ocurre con las mediaciones, vemos que ella aparece en un proceso enfilado más a lograr determinados efectos en aprendizaje, desarrollo, etc., que a orientar ${ }^{4}$ a los sujetos hacia los recursos que se están poniendo en juego para lograr determinados resultados. Incluso, en los casos en que, durante la mediación, se le hace señalamientos al sujeto en desarrollo, acerca de los procesos mediacionales, es norma que no se lleven a cabo tratamientos sistemáticos para la apropiación de la mediación o de la actividad mediadora: recursos de mediación y actividad de mediación, quedan a la sombra, y sus efectos se desencadenan sin que medie la conciencia del sujeto.

Así, en situaciones como aquellas en las que a niños pequeños se les presentan ciertas tareas para comprobar si son capaces de realizarlas con o sin la ayuda o intervención (mediación) de un adulto, se observa que cuando ellos no puedan realizarlas sin ayuda, o su desempeño no alcanza determinados parámetros cuantitativos y/o cualitativos, el adulto que actúa como mediador suele introducir ayudas, orientaciones o andamiajes, que apuntalan la actuación del niño respecto a la tarea. Sin embargo, es de resaltar que en tales situaciones, es habitual que el pequeño permanezca ajeno a que está en una situación donde uno de los factores relevantes es el monto y la naturaleza de la ayuda que se le ofrece, con lo cual la orientación de éste hacia la ayuda permanece en un segundo plano. A partir de lo anterior, no se tienen en consideración circunstancias como que el niño desee o no recibir ayuda; que pueda solicitarla específicamente para algunas partes de la ejecución eliminándola en otras, y así por el estilo.

Como he hecho notar en otras ocasiones (Labarrere, 2003; Labarrere, 2006a), los mecanismos de la acción en las situaciones pedagógicas enfiladas al desarrollo no se abren a la conciencia de los sujetos en desarrollo. Incluso he hecho referencia acerca de que la actuación mediadora del adulto puede entrañar no sólo consecuencias calificables como desarrollo, sino que también puede tener repercusiones en la aparición de cualidades no deseables del comportamiento como la adaptación excesiva a recibir ayuda por parte de los otros, o la emergencia de patrones de interacción que revelan subordinación y falta de iniciativa.

Mi idea es que para todos los procesos de mediación, que pretendan generar desarrollo, el dominio del comportamiento, asociado a la conciencia de la mediación, es básico. En este ámbito pedagógico afirmo que se trata del dominio de las condiciones que generan el desarrollo, o sea, de los procesos mediadores, sus mecanismos y consecuencias.

El dominio de los procesos mediadores es vital para el desarrollo de la autorregulación, la creatividad y, sobre todo, la competencia o habilidad para orientarse y situarse finalidades, aspectos estos tan caros en la denominada sociedad de la información. En este sentido sostengo la necesidad y pertinencia de los aprendizajes de mediación. En este sentido he sostenido que tanto los aprendizajes de orientación, como los aprendizajes de mediación resultan fundamentales para la enseñanza (Labarrere, 2006).

Voy a relatar algunos hechos que apuntan hacia la necesidad de trabajar los aprendizajes de mediación; unido a ello va la idea de que al promover este género de aprendizajes, es necesario abordar la orientación de manera directa.

En una ocasión, hace casi una veintena de años, mi hijo mayor trajo a la casa uno de sus

4 La llamada de atención sobre la orientación como elemento central a tener en consideración no es nueva, buena cantidad de autores ha hecho alusión a que la orientación, la capacidad, habilidad, o dicho en términos más actuales la competencia de orientación, es uno de los componentes principales de la psiquis y del desarrollo del funcionamiento humano. A mi juicio fue un seguidor de Vigotsky (de segunda generación), P. Ya. Galperin, quien, desde las concepciones objetivistas prevalecientes en el momento, la trató como característica esencial de la actividad humana en su dimensión psicológica y pedagógica. 
exámenes de Inglés del preuniversitario, los cuales eran devueltos a los estudiantes luego de revisados por el profesor. Mientras lo analizaba con él me di cuenta de que una de las preguntas contenía, accidentalmente supongo, casi de forma evidente, la respuesta de otra que se hacía un poco más adelante en el mismo examen. Me sorprendió que mi hijo no hubiera reparado en este hecho, lo cual le hubiese permitido contestar sin mucho esfuerzo adicional, o al menos de manera casi directa, la susodicha pregunta. Lo anterior quedó grabado en mí, hasta que al paso de los años tuve la posibilidad de abordarlo como objeto de investigación.

A partir de lo anterior, comencé una línea de pensamiento en el sentido de estudiar el aprovechamiento de la ayuda introducida en diversas situaciones (solución de problemas, los exámenes, etc.), así como el grado de atención y conciencia que los estudiantes muestran ante dicha ayuda. Obviamente, al abordar el fenómeno aludido, estoy aproximándome a lo que puede denominarse como toma de conciencia o aprendizaje de la mediación.

En este sentido, he abordado dos fenómenos: 1) los procesos de alertamiento y orientación ante la ayuda "indirectamente" introducida en las situaciones y, 2) la potencialidad de las situaciones e "instrumentos" pedagógicos para generar acciones de orientación y exploración por parte del estudiante; en particular las situaciones de evaluación de los aprendizajes. En esta dirección he trabajado con lo que denomino situación evaluativo-experimental (docente-experimental).

De manera general he podido establecer que dichos instrumentos (pruebas, problemas y tareas, etc.) por lo común se asumen sin más ni más, sin que medie la necesaria actividad exploratoria. Son instrumentos para ejecutar, problemas para resolver, pautas para seguir y prácticamente nunca algo para investigar.

En la primera dirección, trabajé, hace tiempo, según las clásicas tareas que aparecen en A Study of thinking de Bruner (1956), relativas a la formación de conceptos y el desarrollo de estrategias (con alumnos de nivel medio). Diseñé una tarea en cuyas instrucciones deslicé, como sin querer, una orientación hacia las estrategias. Concretamente la situación experimental fue la siguiente. $\mathrm{Al}$ ofrecer la consigna les decía que por lo general al enfrentarse a la solución de problemas y la realización de tareas, las personas utilizan estrategias. Definí la estrategia y hablé de las variaciones que solían experimentar y de que las personas podían ser o no conscientes de los modos en que abordan las actividades que realizan. Todo esto con la finalidad de comprobar si la orientación que contenía alusión a las estrategias, originaba en los estudiantes alertamiento y que, consecuentemente, prestaran atención a su comportamiento durante la ejecución de la tarea; es decir tratando de favorecer la emergencia de los procesos de orientación, monitoreo y control de la conducta, abordados por la metacognición. Puede verse que estaba situando directamente a los estudiantes la tarea de resolver un problema y, a la vez generando la posibilidad de que ellos se orientaran a la exploración de su conducta, pero esto último de manera indirecta; por tanto, que ofrecieran relevancia a las estrategias como vía para organizar su comportamiento.

Los resultados mostraron que la referencia a las estrategias y al comportamiento humano en condiciones de resolver problemas, que podía verse como cierto recurso a la mediación en su sentido más amplio de generar orientación, no funcionaba; es decir el grado de orientación hacia esa parte de las indicaciones era nulo. Así por ejemplo, durante la administración de la consigna experimental, comprobé que los estudiantes nunca hacían preguntas en torno a la consigna, no era tomada como algo para investigar en aras de un mejor desempeño. Es fácil colegir que la ausencia de orientación y elaboración explícita de la consigna, forma parte del género e conductas pasivas que han sido estampadas en los alumnos por una enseñanza normativa, no indagadora y cuestionadora, con lo cual el estudiante ha devenido un ser pasivo respondiente, lleno de autobloqueos y autolimitaciones que penetran muy hondo, a veces imperceptiblemente, en su desempeño docente y desenvolvimiento como ciudadano.

Además, logré comprobar la relativa ausencia de acciones dirigidas a monitorear su comportamiento. En una segunda parte de la investigación, para asegurarme acerca de si los estudiantes tenían o no conciencia de la estrategia, les pedí que modificaran la estrategia que 
habían seguido y que emplearan otra diferente. Cuando se les solicitaba el cambio de estrategia, los estudiantes reaccionaban con extrañeza y se notaba que durante la ejecución de la tarea se habían centrado más en hallar la solución, que en estudiar el proceso que seguían. Obviamente el despliegue metacognitivo estaba en déficit. Al pedirles que cambiaran la estrategia, ellos se orientaban hacia la actividad y generaban toda suerte de modificaciones que por lo común eran inconsistentes, desorganizadas y, sobre todo, carecían de sustentación en explicaciones coherentes; por lo que era lógico pensar que se originaban in situ, como una respuesta de adaptación a las exigencias que les hacía, pero nada más.

Así, comprobé que no tenían referencia, ni conciencia de la estrategia, con lo cual se puede decir que la mediación, introducida de manera indirecta, sin trabajar en la conciencia y la orientación hacia la misma, no provocaba realmente orientación metacognitiva en los estudiantes. De lo anterior se deriva no sólo la ausencia de actividad metacognitiva en los estudiantes, sino también que para trabajar el aprendizaje estratégico o de estrategias es necesario abordar explícitamente los procesos orientadores.

A partir de los resultados que obtuve, continué empleando la introducción de ayudas indirectas en situaciones para explorar los procesos de orientación de los estudiantes. Avanzando en el mismo sentido, el segundo grupo de investigaciones, se refiere a los procesos de orientación, análisis y empleo (conciencia e intencionalidad) con que los estudiantes universitarios procesan pistas deslizadas, también como al azar, en diferentes situaciones evaluativas (exámenes). He utilizado un procedimiento que consiste en ayuda contextual indirecta, la ayuda contextual indirecta se efectúa mediante la introducción en preguntas u orientaciones de exámenes, información que potencialmente facilita el análisis de los estudiantes o que sitúan, de manera indirecta, determinados requisitos para la acción consecuente al contestar otras preguntas.

Partí del hecho de que la conducta inteligente durante la realización de pruebas y exámenes, consiste en buscar apoyos en las propias situaciones e instrumentos, como ayudas para responder las preguntas; pienso que en cada situación de examen se pueden encontrar claves para las respuestas, sobre todo si, como dice Ausubel (Meaninful Learnig), existe una estructura lógica, lo que implica la presencia de una organización lógica subyacente a los tópicos, que incluso suele escapar a quienes diseñan las pruebas y los exámenes. Con este criterio en mente, en varias ocasiones he introducido ayudas en los exámenes; por ejemplo, una pregunta que contiene la respuesta a otra del temario (siguiendo la lógica que me sugirió el suceso más arriba relatado); una indicación para resolver una pregunta, etc.

Por ejemplo, en una prueba que tenían que hacer estudiantes de psicología, en una pregunta que aparecía como teórica, les solicitaba que manifestaran su punto de vista en torno a posiciones diferentes en el análisis de los fenómenos (una orientación aislacionista que ve los sucesos por separado, sin interconexión, y otra orientación sustentada en la complejidad, que reconoce la no separación y la constitución al unísono). Más adelante en la prueba, otra pregunta solicita el análisis de una situación donde se muestran determinadas relaciones (algunas indicadas por las habituales flechas con doble saeta y otras flechas que indican un solo sentido). Se trata de ver entonces, la consecuencia entre las respuestas a una y otra pregunta y, sobre todo, si lo que en la primera ellos plantean como relevante, se aplica en la otra pregunta y si les sirve como indicador para actuar en consecuencia.

De manera consistente, he podido comprobar que estas ayudas o mediaciones contextuales indirectas, resultan ineficaces por varias razones, entre las cuales se halla el hecho de que los estudiantes no les prestan atención, debido a que ante las situaciones evaluativas (pruebas, exámenes, etc.) realizan un análisis parcial de las mismas, despliegan orientaciones superficiales y prevalece la tendencia a trabajar en un plano esencialmente instrumental operativo (Labarrere, 1998; Labarrere y Quintanilla, 2002). Así, los resultados indican, de forma consistente, la ausencia o déficit en la orientación, exploración e investigación de las situaciones, específicamente en lo que se refiere a la búsqueda sistemática de apoyos y en la elaboración activa de mediaciones que pudieran ser eficaces para el enfrentamiento y la solución de las tareas y problemas. 


\section{De los aprendizajes para la mediación y el necesario tránsito de la ayuda a la colaboración.}

Desde mi punto de vista, en la base de los aprendizajes de mediación y para la mediación, se halla lo que he denominado "Transparencia metacognitiva” (Tmc). Esta noción la veo como los intentos conscientes de los sujetos por revelar y penetrar (poner de relieve) los respectivos "códigos ocultos” de las actividades; son esfuerzos conscientes por dar significado a los qué, cómo, para qué etc., de los procesos de mediación pedagógica. Además de la Tmc, los aprendizajes de mediación requieren o aluden a un conjunto de aspectos, entre los cuales yo identifico:

a) Disposición favorable a explorar la mediación (ayuda, andamiaje, etc.),

b) Conocimiento acerca de que la actividad suele ser mediada y automediada,

c) Identificación de situaciones de mediación,

d) Generación de oportunidades de mediación para la actividad de los otros y para la propia.

La apropiación de los mecanismos de la actividad mediadora, es un elemento crucial en lo que denomino necesario tránsito de la ayuda a la colaboración, como eje e indicador del desarrollo. En varias ocasiones he afirmado que si para Vigotsky, lo que el sujeto en desarrollo debe hacer mañana, sin ayuda, es resolver el problema que hasta ayer solo resolvía con ayuda, y esto un indicador apropiado del verdadero desarrollo; en mi criterio lo que el sujeto debe realizar mañana solo, no es tanto, o solamente, resolver el problema sin ayuda; como poder ser capaz de desplegar mecanismos de autoandamiaje, tanto en lo personal, como en las situaciones que requieran que él ofrezca ayuda a los otros. Esto es sumamente importante, en vista de la interacción entre los estudiantes, fundamentalmente el trabajo en equipos y las situaciones de tutoría de un estudiante a otro. La posibilidad de autoayuda significa el logro del control del comportamiento a través del dominio de las condiciones que generan o promueven el comportamiento mismo expresado en la posibilidad creciente de intervenir en procesos de desarrollo, con conciencia.

Pero no solamente los aprendizajes de mediación son importantes para la interacción entre los estudiantes; sino también para la interacción con los profesores. Creo que paulatinamente la interacción profesor-alumno debe irse transformando en interacción-colaboración profesor alumno; de ahí que he postulado que uno de los objetivos principales de la acción en ZDP lo constituye el logro del tránsito de la ayuda a la colaboración; de donde mi formulación de ZDP, se inclina a considerarla como el lugar donde se producen tránsitos de la ayuda a la colaboración bajo la acción mediada. El tránsito se facilita, precisamente, por los aprendizajes de mediación antes referidos. La necesidad de este tránsito resulta más evidente cuando se trata de la formación profesional y, más aún, cuando se trata de la formación de profesores; en tales situaciones, los aprendizajes de mediación, la apropiación de los mecanismos de la acción mediadora que se aplican a los propios procesos formativos que están transcurriendo "aquí y ahora”, constituyen buena parte de la piedra angular del desarrollo de los profesionales en formación (Labarrere, 2003a). Pero toda esta historia debe comenzar mucho antes, durante la formación básica y media.

Todo lo que he planteado implica y sitúa la posibilidad de transitar de la ayuda a la colaboración en los procesos mediadores. Creo que en general, “de la ayuda a la colaboración” debería ser la divisa de estos tiempos en la enseñanza, la formación de las nuevas generaciones; ofrecernos la posibilidad de mediar para la vida mediante una colaboración respetuosa y abierta.

Para mí, el tránsito hacia la automediación y hacia una cultura de la mediación señala el camino del desarrollo. Es un tránsito no sólo instrumental y cognitivo, sino también en la responsabilidad. El tránsito que señalo tiene concomitantes éticos profundos, entre ellos los nuevos roles, atribuciones y responsabilidades que estarían en manos de los estudiantes. Considerar a los estudiantes más como colaboradores que como simples alumnos, puede llevar a transformar la ética de la formación, a generar nuevos desafíos para profesores y estudiantes, nuevas miradas que se tiendan sobre los sujetos de la formación.

No sé en qué medida estemos preparados para ese paso trascendental, no sólo los profesores, sino 
los propios estudiantes. De cualquier forma deberíamos darlo. Tal vez ya estemos en camino.

\section{Referencias}

Bruner, J. (1956). A Study of thinking. New York: Wiley.

Cole, M. (1989). Cultural Psychology: A once and future discipline? En J.J. Berman (Ed.), Nebraska Symposium on Motivation, Cross-cultural perspectives, 37 (pp. 279-336). Lincoln: University of Nebraska Press.

Galperin, P. (1982). Introducción a la psicología. La Habana: Pueblo y Educación.

Labarrere, A. (1995). Pensamiento: el análisis y la autorregulación en la actividad cognoscitiva de los estudiantes. México: Ángeles Eds.

Labarrere, A. (2003). Funcionamiento Cognitivo y Desarrollo en ZDP. Pensamiento Educativo, 32,141154.

Labarrere, A (2003a). La formación de profesores de ciencia: un enfoque desde la teoría de la profesionalización temprana y de los procesos de orientación. Revista Extramuros $N^{\circ}$ 2, 21-33.

Labarrere, A. (2006). Aprendizaje, complejidad y desarrollo: Agenda curricular para enseñar en los tiempos actuales. Revista de Psicología Universidad de Chile, XV, (2), 65-76.

Labarrere, A. (2006a). Interacción en la Zona de Desarrollo Próximo-ZDP: ¿Qué puede ocurrir para bien y qué para mal? En Internet: http://www.ciberdocencia.gob.pe/index. php?id=379\&a=articulo_completo abril 2006.

Labarrere, A. y Quintanilla, M. (2002). La solución de problemas en el aula. Reflexiones sobre los planos de análisis y desarrollo. Pensamiento Educativo, 30, 121-138.

Lombart, M. y Ditton, T. (2008). At the Heart of It All: The Concept of Presence. En Internet: http:// jcmc.indiana.edu/vol3/issue2/lombard.html Extraído: Noviembre de 2008.

Noguez, S. (2006). El desarrollo potencial de aprendizaje. Entrevista a Feuerstein. En Internet: http://64.233.161.104/search?q=cache:zJ0ZXdHd_6oJ:redie.uabc.mx/vol4no2/contenidonoguez.pdf+feuerstein+entrevista\&hl=es\&gl=cl\&ct=clnk\&cd=1 , extraído octubre 2006.

Ratner, C. (1991). Vygotsky's sociohistorical psychology and its contemporary applications. New York: Plenum Press.

Vigotysky, L. (1987). Historia del desarrollo de las funciones psíquicas superiores. La Habana: Editorial Científico Técnica. 